\title{
Evaluation of LoRa LPWAN Technology for Indoor Remote Health and Wellbeing Monitoring
}

\author{
Juha Petäjäjärvi ${ }^{1}$, Konstantin Mikhaylov ${ }^{1}$, Rumana Yasmin ${ }^{2}$, Matti Hämäläinen ${ }^{1}$, \\ and Jari Iinatti \\ ${ }^{1}$ Centre for Wireless Communications, University of Oulu, \\ Erkki Koiso-Kanttilan katu 3, 90570 Oulu, Finland \\ \{firstname.lastname\}@ee.oulu.fi \\ ${ }^{2}$ University of Oulu, Pentti Kaiteran katu 1, 90014 Oulu, Finland \\ \{firstname.lastname\}@ student.oulu.fi
}

\begin{abstract}
Long lifetime of a wireless sensor/actuator node, low transceiver chip cost and large coverage area are the main characteristics of the low power wide area network (LPWAN) technologies. These targets correlate well with the requirements imposed by the health and wellbeing applications of the digital age. Therefore, LPWANs can found their niche among traditional short range technologies for wireless body area networks, such as ZigBee, Bluetooth and ultra wideband. To check this hypothesis, in this work we investigate the indoor performance with one of the LPWAN technologies, named LoRa, by the means of empirical measurements. The measurements were conducted using the commercially available devices in the main campus of the University of Oulu, Finland. In order to obtain the comprehensive picture, the experiments were executed for the sensor nodes operating with various physical layer settings, i.e., using the different spreading factors, bandwidths and transmit powers. The obtained results indicate that with the largest spreading factor of 12 and $14 \mathrm{dBm}$ transmit power, the whole campus area (570 meters North to South and over 320 meters East to West) can be covered by a single base station. The average measured packet success delivery ratio for this case was $96.7 \%$, even with no acknowledgements and retransmissions used. The campus was covered also with lower spreading factors with $2 \mathrm{dBm}$ transmit power, but considerably more packets were lost. For example with spreading factor $8,13.1 \%$ of the transmitted packets were lost. Aside of this, we have investigated the power consumption of the LoRa compliant transceiver with different physical layer settings. The experiments conducted using the specially designed module show that based on the settings used, the amount of energy for sending the same amount of data may differ up to 200 -fold. This calls for efficient selection of the communication mode to be used by the energy restricted devices and emphasizes the importance of enabling adaptive data rate control.
\end{abstract}

Keywords: IoT, chirp spread spectrum, spreading factor, RSSI, coverage, range, power consumption. 


\section{Introduction}

The enablement of long range, low cost and, at the same time, energy efficient wireless communications introduces a lot of new possibilities for the novel applications in the diverse application domains. This has driven a lot of attention to the recently introduced low-power wide area network (LPWAN) technologies from the industrial and the academic communities. The landscape of the LPWAN technologies today is really diverse and spans over a variety of the competing technologies, such as Sigfox, LoRa, 3GPP originating LTE-M and NB-IoT, Weightless, Ingenu, Waviot, nWave, Telensa, Cyan's Cynet, Accellus, and SilverSpring's Starfish [1]. Aside of the conventional application areas, such as infrastructure monitoring and metering scenarios (e.g., gas, water or electricity consumption metering), as well as asset tracking and smart traffic, the LPWANs can become the enablers for new applications in other fields, one of them being the smart healthcare and wellbeing monitoring.

Traditionally, the personal healthcare systems have been designed based on the wireless body area networks (WBANs), which typically consist of wearable sensor nodes equipped with short range radios, and communicating with a gateway having a connection to a backbone network [2]. The use of the conventional short range communication technologies limits the distance between the gateway and the WBAN sensors to tens of meters depending on the technology used and the environment of operation [3]. To ensure connection between sensor nodes and the gateway, conventionally, the gateway has also been a wearable device, such as, a smartphone. The LPWAN technology enables to omit the gateway device. Namely, the LPWANs extend the communication distances between the sensors and the base stations to hundreds of meters or even tens of kilometers long. On one hand, this will result in reduction of the cost thus making such solutions more affordable. Also this substantially limits the complexity of these networks, enabling in most cases to utilize a simple star network with an LPWAN base station in the center. To give a practical example, one can consider remote monitoring of patients at hospitals or even their accommodations located near the hospitals by deploying a single base station covering the whole facility and the nearby city district.

Aside of simplifying the network's topology and waiving the respective costs, the use of LPWANs in human-centric applications may bring other benefits. The LPWAN technologies are typically designed paying special attention for achieving very low cost and low energy consumption for the sensor devices. Both of these features are of significant importance, also for the smart healthcare and wellbeing applications [4, 5]. Nonetheless, there are some drawbacks using LPWANs in these monitoring applications due their natural limitations. First of all, LPWANs typically assume highly asymmetric communication link with the prevalence of uplink traffic. The downlink is primarily used for acknowledgements, whereas uplink is being intensively utilized for transmitting the measured data. Second, in order to achieve the wide area coverage, the over-the-air data rate has been decreased to kilobits per second or even less than that. This may result in substantial delays in the data transfer. Third, in order to minimize the "radio on" time and the respective power consumption, the existing LPWAN 
technologies often rely on very simple media access mechanisms (e.g., Aloha). Given that these technologies often operate in the license-free and already congested industrial, scientific and medical (ISM) bands, this may compromise the reliability of such links. Finally, the operation in the sub-GHz ISM bands imposes restrictions on the linear dimensions of, e.g., the antenna system, which may not be easily satisfied in the miniature wearable devices. All the said above raises the question of to which extend and for which applications can the LPWANs be used in the context of smart healthcare and wellbeing? This is the fundamental question which we attempt to address in this paper.

Although the LPWAN technologies can hardly be treated as an ultimately new development (e.g., Sigfox founded in 2009), they were nearly ignored by the academia so far. One of the major reasons for this is the fact that the development of these technologies has been primarily driven by the industry and not so much accurate data have been made available. Nonetheless, today, with the appearance of the commercial hardware and the sheer deployment of the LPWAN infrastructure, the situation starts to change. An overview of nine different LPWAN technologies has been reported in [6]. An overview of the LoRa technology is introduced in [7]. Detailed description of the physical layer and medium access control of the two LPWAN technologies, the deployment of which is currently actively ongoing, namely Sigfox and LoRa, has been presented in [8]. In our previous studies we have focused on the different aspects of the LoRa LPWAN technology and reported the results for the technology scalability and capacity analysis in [9], the outdoor coverage measurements and channel characterization in [10], and the effect of mobility and Doppler robustness on communication performance in [11]. In [12] we have initially proposed the possibility of employing the LoRa LPWAN technology for wearables and have reported the first results, which are expanded further in this paper.

In this paper, we study the performance of the LoRa LPWAN technology in non-line-of-sight (NLOS) indoor environment in general and the human wellbeing monitoring applications in particular. The paper summarizes the results of an extensive experimental measurement campaign conducted using the commercial LoRaWAN [13] compliant devices in the main campus area of the University of Oulu, Finland. In the studies we have experimentally investigated the effect of the different communication mode settings defined in the LoRaWAN specification for the EU 863-870 MHz band on the communication performance. To the best of our knowledge, this is the first attempt in open literature to comprehensively study the indoor performance of the technology. Aside of this, we report the results revealing the power consumption of a LoRaWAN transceiver in the various modes, which have been measured using the prototype of our LoRaWAN-enabled sensor device. The reason why we have selected LoRa for our study is twofold. First, this is one of the few LPWAN technologies that are today commercially available. Second, the use of the license-free ISM band and the availability of the base station for purchase enables any third party or even an individual to deploy an LPWAN. On one hand, this alleviates many difficulties related to the experimental study of this technology. On the other hand, this facilitates trialing and developing the various practical applications based on this communication technology. 
This paper is organized as follows. Section 2 introduces the major technical features of the LoRa technology. Section 3 describes the measurement setup for the indoor coverage measurements. The results are reported and discussed in Section 4. Section 5 concludes the paper and summarizes the obtained results.

\section{The LoRaWAN Technology}

The version 1 of the LoRaWAN specification [13] addresses several different aspects of the technology, ranging from the physical and link layers up to the network architecture.

\subsection{Physical layer}

In Europe, LoRaWAN operates in the $863 \mathrm{MHz}-870 \mathrm{MHz}$ the ISM band. There is also option for $433 \mathrm{MHz}$ ISM band, which enables even longer communication distances. ISM bands are also used in the United States and in China, where the operation frequencies are $902 \mathrm{MHz}-928 \mathrm{MHz}$ and $779 \mathrm{MHz}-787 \mathrm{MHz}$, respectively. [13]

In the EU frequency bands, the LoRaWAN physical layer supports two different modulation schemes. The first one is the proprietary LoRa modulation based on chirp spread spectrum (CSS) technique. In CSS, the frequency of the carrier continuously increases or decreases linearly following the chirp signal covering the whole available bandwidth $(B W)$. The use of spectrum spreading introduces processing gain for the received CSS radio signal in the receiver, which improves the link budget. The possibility of changing the spreading factor $(S F)$ enables trading the on-air time for the range of communication. For the maximum $S F$ (i.e., 12) the link budget for LoRa modulation with $20 \mathrm{dBm}$ transmit power reaches $157 \mathrm{~dB}$, which enables multi-kilometer communication range or, alternatively, can enable reducing the transmit power. The effective on-air bit rate is calculated as [14]

$$
R_{\mathrm{b}}=S F \times \frac{1}{\left(\frac{2^{S F}}{B W}\right)} \times C R,
$$

where $B W$ is the bandwidth $C R$ is the code rate. For LoRaWAN in the EU bands the former can take the value of either $125 \mathrm{kHz}$ or $250 \mathrm{kHz}$ and the latter is fixed at $4 / 5$.

The Gaussian frequency shift keying (GFSK) modulation with $50 \mathrm{kbit} / \mathrm{s}$ rate is also supported for applications requiring higher data rates and operating over substantially shorter ranges.

The bit rates and receiver sensitivities for the different modulation methods and $S F$ s available for the EU $868 \mathrm{MHz}$ band are summarized in Table 1. Even though the on-air rates are not very high, the signals with different $S F$ s are orthogonal and thus can be transferred simultaneously in synchronous transmissions (as long as the received power is not significantly higher in one signal than in others that causes near-far problem to occur [15]). This positively affects the spectrum efficiency and the scalability of the 
resulting network. As we have shown in [9] a single LoRaWAN cell can theoretically serve up to several millions of infrequently reporting sensors or few thousands of devices streaming their data. It is also worth noting that in the LoRaWAN specification the different modulation coding schemes (which are listed Table 1) are referred to as Data Rates (DR) and further in this paper we will follow this notation.

Table 1. LoRaWAN data rates based on the different modulations and configuration parameters which are available for the EU $868 \mathrm{MHz}$ band.

\begin{tabular}{lcccc}
\hline Modulation & Spreading factor & Bandwidth & Air bit rate [kb/s] & Sensitivity [16] \\
\hline LoRa & 7 & $125 \mathrm{kHz}$ & 5.468 & $-124 \mathrm{dBm}$ \\
LoRa & 7 & $250 \mathrm{kHz}$ & 10.936 & $-122 \mathrm{dBm}$ \\
LoRa & 8 & $125 \mathrm{kHz}$ & 3.125 & $-126 \mathrm{dBm}$ \\
LoRa & 9 & $125 \mathrm{kHz}$ & 1.757 & $-129 \mathrm{dBm}$ \\
LoRa & 10 & $125 \mathrm{kHz}$ & 0.976 & $-132 \mathrm{dBm}$ \\
LoRa & 11 & $125 \mathrm{kHz}$ & 0.537 & $-134.5 \mathrm{dBm}$ \\
LoRa & 12 & $125 \mathrm{kHz}$ & 0.293 & $-137 \mathrm{dBm}$ \\
FSK & - & $150 \mathrm{kHz}$ & 50 & $-122 \mathrm{dBm}$ \\
\hline
\end{tabular}

Time dispersion due to multipath can be a problem in wireless communications if not taken care of. In multipath propagation, a receiver gets multiple time delayed copies of the target signal that have travelled via the different paths from the transmitter to the receiver. This causes transmitted signal to undergo either flat or frequency selective fading [15]. LoRa technology fights against multipath by using relatively wideband signal in combination with forward error correction (FEC) [17]. Furthermore, LoRa employs random frequency hopping spread spectrum (FHSS) to couple with interference.

\subsection{Medium access and network architecture}

The LoRaWAN networks are laid out in a star-of-stars topology with base stations relaying the data between the sensor nodes and the network server. Communication between the sensor nodes and the base stations goes over the wireless channel utilizing one of the physical layers discussed above, whilst the connection between the gateways and the central server should be handled over a backbone IP-based network.

The LoRaWAN specification defines three classes for sensor nodes to access the medium. All the sensor nodes are required to implement the Class A functionality, and the implementation of Classes B and C functionality are optional. The devices of class A are entitled to start transmitting their packets at any moment of time in Aloha-fashion [18]. The channel to be used for packet's transmission is selected randomly from the pool of channels listened by the base station. Importantly, since no listen before talk technique is used for channel access, the sensor node has to track its on-air time and obey the duty cycle restrictions imposed by the frequency regulations. After sending a frame, the sensor node opens two receive windows, which can be used by the base station to reach the device in downlink. In addition to these windows, the devices of class B have additional periodic receive slots. The class $\mathrm{C}$ devices stay in receive mode 
all the time unless they are transmitting in order to minimize the latency at the cost of higher energy consumption.

It is worth noting that in LoRaWAN the sensor nodes are not connected to any particular base station and a packet sent by the sensor node can be received by multiple base stations within node's communication range. Thus, specific handover procedures are not needed eliminating the handover related signaling, which brings energy savings to the sensor nodes and increases the probability of successful packet delivery. [19]

The network server is responsible for security, diagnostics, filtering redundant packets, acknowledgements, and optimizing data rate for static sensor nodes. The latter is handled with an optional adaptive data rate (ADR) feature. If this feature is enabled, the network controls the DRs used by the individual EDs. To give a practical example, if network detects that the power of an uplink packet received by a base station is strong enough, it can command the sensor node to switch to lower $S F$. This enables energy savings for the sensor nodes, since packets are faster to transfer with lower $S F$. [19]

\section{$3 \quad$ Measurement setup and experimental environment}

The measurement campaign for this paper was done in two phases. The first phase took place during autumn of 2015 and the second phase during summer 2016. The measurements were conducted in the Linnanmaa campus at the University of Oulu, Finland. The campus area is more than 570 meters North to South and over 320 meters East to West. It consists of numerous buildings that are connected together by indoor passages. The building frames are mostly built of concrete and steel.

In the first measurement phase, a battery-powered sensor node was attached to a researcher's arm with a band, as shown in Fig. 1. A plastic sheet was inserted between clothing and an antenna to prevent antenna from touching the body or the clothes since this could have caused a negative impact to its performance. During the experiments, the researcher followed his daily routine. Thus, the researcher acted accordingly in each location (e.g., took the food from the line, pay it, and ate at a table at the restaurant). The researcher walked between different locations with a typical walking speed of about 5 $\mathrm{km} / \mathrm{h}(1.4 \mathrm{~m} / \mathrm{s})$. The measurement locations are shown in Fig. 2. This phase was intended to evaluate the feasibility of using the LoRaWAN LPWAN technology for human-centric applications. The initial results of this study have been reported in [12].

Since the initial studies proved that the LoRaWAN is capable of operating in indoor environment, during the second phase of the measurement we have conducted a set of experiments intended to characterize the effect of the communication settings on the performance and reliability of LoRaWAN-based communication. For these measurements we have employed a sensor node powered from a laptop via a USB-cable. 


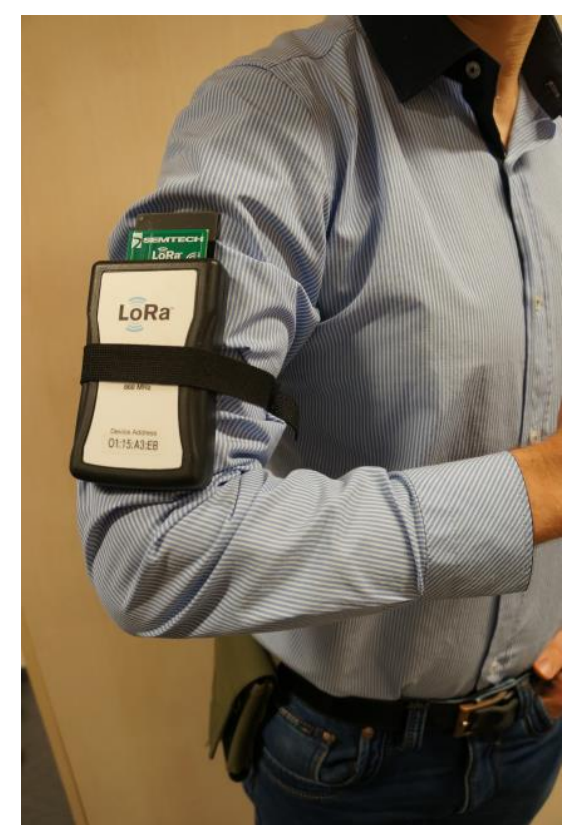

Fig. 1. The sensor node attached to researcher's arm during measurement phase one.

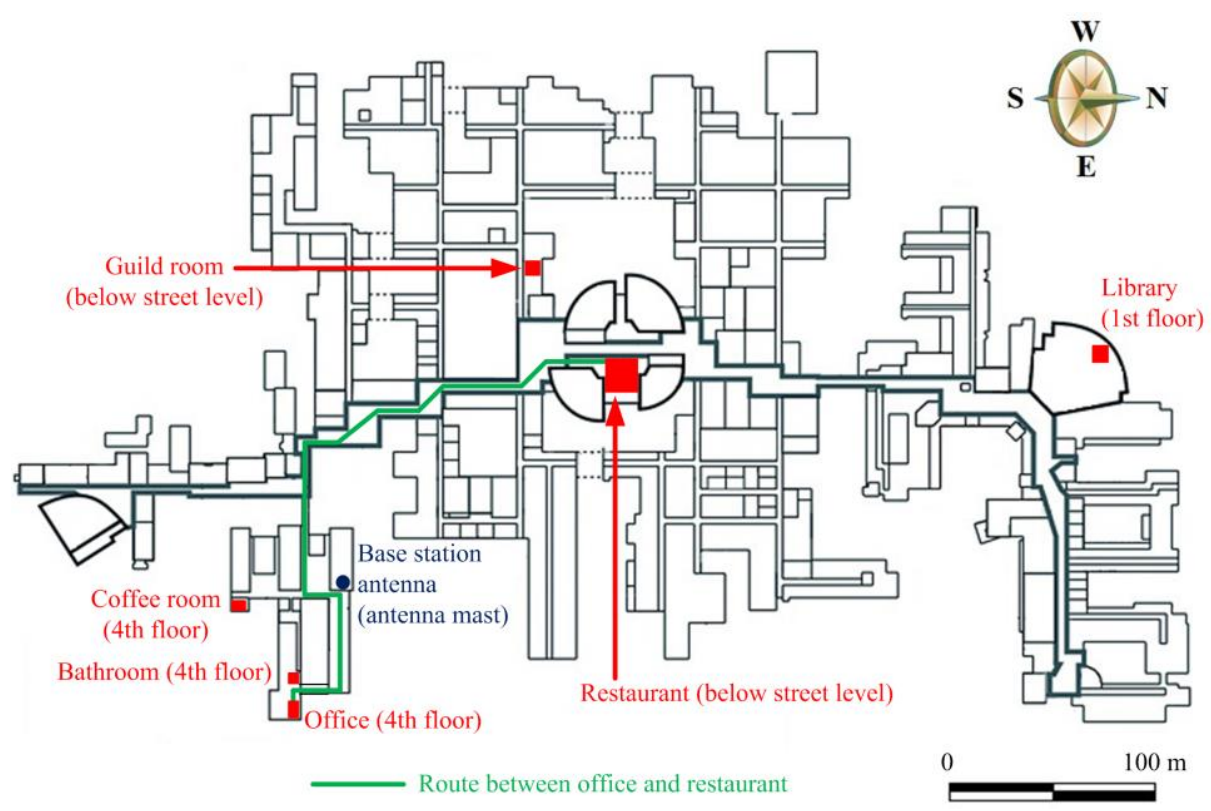

Fig. 2. First phase measurement locations at the University of Oulu. 


\subsection{The sensor node}

The LoRaMote [20] sensor node used in the measurements is built around the SX1272 transceiver from Semtech [15], and connected to a planar-F type printed circuit board antenna. Since the information about the indoor performance of the LoRa technology prior to our measurements was very scarce, for the first phase we have configured the sensor node to use the largest possible $S F=12$ with $B W=125 \mathrm{kHz}$, and the transmit power was fixed at $14 \mathrm{dBm}$. In this case, the sensitivity of the receiver is maximum and reaches $-137 \mathrm{dBm}$, giving the total radio link budget of more than $150 \mathrm{~dB}$. For the second set of measurements the transmit power was reduced to $2 \mathrm{dBm}$, which is the minimum allowed by the specification. Coding rate was set to $4 / 5$ as prescribed in the LoRaWAN specification [13]. In the second phase of the measurements we have varied the DR used by the node.

During the measurements the sensor node was configured to send a message to the base station every five seconds. However, the restrictions on duty cycle imposed by the EU radio frequency regulations in the $868 \mathrm{MHz}$ ISM band [21] $(0.1 \%$ to $10 \%$, in different bands) resulted in longer delays between the packets. The sensor node was entitled to use the six different channels that are listed in Table 2. The radio transceiver switched between the transmission channels automatically, calculated the time of use for each of the channels and accounted for the restrictions imposed by the frequency usage regulations. The regulations are listed in two rightmost columns in Table 2. Neither the acknowledgements or retransmissions, nor the ADR were enabled during the measurement campaigns.

Table 2. Utilized frequency bands and regulations for them.

\begin{tabular}{lcc}
\hline Center frequency & Maximum allowed transmit power [21] & Spectrum access [21] \\
\hline $868.100 \mathrm{MHz}$ & $14 \mathrm{dBm}$ & $1 \%$ or LBT AFA \\
$868.300 \mathrm{MHz}$ & $14 \mathrm{dBm}$ & $1 \%$ or LBT AFA \\
$868.500 \mathrm{MHz}$ & $14 \mathrm{dBm}$ & $1 \%$ or LBT AFA \\
$868.850 \mathrm{MHz}$ & $14 \mathrm{dBm}$ & $0.1 \%$ or LBT AFA \\
$869.050 \mathrm{MHz}$ & $14 \mathrm{dBm}$ & $0.1 \%$ or LBT AFA \\
$869.525 \mathrm{MHz}$ & $27 \mathrm{dBm}$ & $10 \%$ or LBT AFA \\
\hline
\end{tabular}

${ }^{1}$ Listen before talk adaptive frequency agility (LBT AFA)

\subsection{The base station}

As a base station, we have used the Kerlink's LoRa IoT station, which was installed in the premises of the Faculty of Information Technology and Electrical Engineering. The base station was connected to the university's local network, which was used as a backbone to transfer the collected data to the network server and a data base run by Semtech. The antenna of the base station was mounted at the University of Oulu antenna tower at the height of approximately $24 \mathrm{~m}$ from sea-level. The antenna is the biconical D100-1000 from Aerial [22] and it provides 2 dBi gain (shown in Fig. 3). The base station was configured to listen at the six frequency channels, which the sensor node used for its transmissions (see Table 2). 
Each of the packets received by the base station was stored as a separate entry in the data base, listing aside of the actual payload the sequential number of the packet, the timestamp, the received power, as well as the information about the DR and the frequency channel used for this packet. Data has been downloaded from the data base and analyzed further.

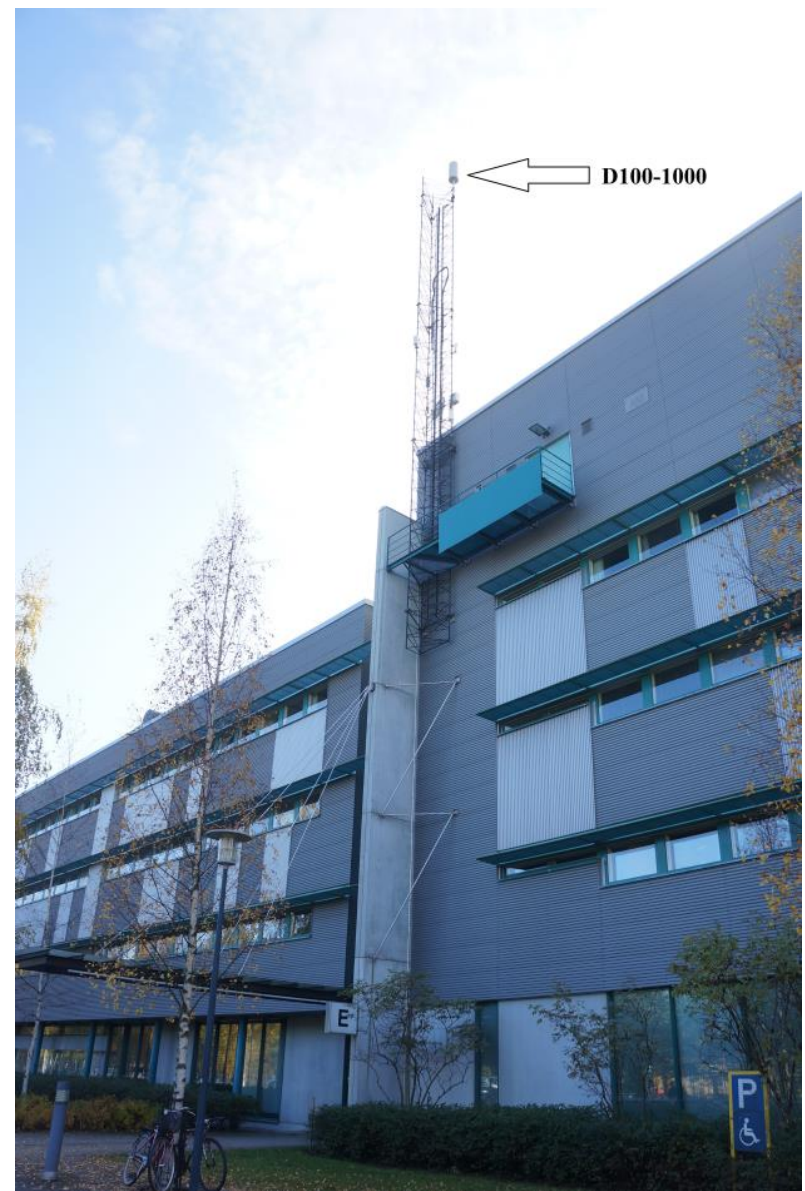

Fig. 3. The antenna is mounted on the University of Oulu antenna tower at a height of $24 \mathrm{~m}$ from sea level.

\section{$4 \quad$ Results}

In this Section we first present the results of the first phase indoor coverage measurements conducted using the maximum $S F$. Then we discuss the results of the measurements executed with the other LoRaWAN physical layer settings. Finally, in Section 4.4, we also present the results of the power consumption measurements for the 
LoRaWAN compliant transceiver with different $S F$ s and transmit powers.

\subsection{Coverage with spreading factor 12 and $125 \mathrm{kHz}$ bandwidth}

The results of the measurements are summarized in Tables 3-5. Tables 3 and 4 list the estimated distance between the base station's antenna and each measurement location, as well as the respective number of transmitted and received packets and the calculated packet error ratio for each location and mobile scenarios. The results show that in average $96.7 \%$ of all the data packets were successfully transferred for all the locations. When the target was moving, the success rate was about $95 \%$.

The base station recorded received power for each packet. This information together with transmit power $(14 \mathrm{dBm})$, and the antenna gain $(2 \mathrm{dBi})$ enabled us to calculate the path loss for each received packet. The average path loss and the standard deviation for the different locations are shown in Table 5.

Table 3. Packet error rate from different locations $(S F=12$, transmit power $=14 \mathrm{dBm})$.

\begin{tabular}{lcccc}
\hline Location & $\begin{array}{c}\text { Distance to } \\
\text { the BS }\end{array}$ & $\begin{array}{c}\text { No. of Tx } \\
\text { packets }\end{array}$ & $\begin{array}{c}\text { No. of Rx } \\
\text { packets }\end{array}$ & $\begin{array}{c}\text { Packet error } \\
\text { rate }\end{array}$ \\
\hline Office (4th floor) & $65 \pm 5 \mathrm{~m}$ & 1796 & 1758 & $2.1 \%$ \\
Bathroom (4th floor) & $54 \pm 2 \mathrm{~m}$ & 331 & 329 & $0.6 \%$ \\
Coffee room (4th floor) & $52 \pm 5 \mathrm{~m}$ & 736 & 717 & $2.6 \%$ \\
Main restaurant (below street level) & $180 \pm 30 \mathrm{~m}$ & 1245 & 1193 & $4.2 \%$ \\
Main library (1st floor) & $390 \pm 30 \mathrm{~m}$ & 878 & 831 & $5.3 \%$ \\
Guild room (below street level) & $195 \pm 15 \mathrm{~m}$ & 340 & 322 & $5.3 \%$ \\
Total & - & 5326 & 5150 & $3.3 \%$ \\
\hline
\end{tabular}

Table 4. Packet error rate under target mobility $(S F=12$, transmit power $=14 \mathrm{dBm})$.

\begin{tabular}{lcccc}
\hline Location & $\begin{array}{c}\text { Distance to } \\
\text { the BS }\end{array}$ & $\begin{array}{c}\text { No. of Tx } \\
\text { packets }\end{array}$ & $\begin{array}{c}\text { No. of Rx } \\
\text { packets }\end{array}$ & $\begin{array}{c}\text { Packet error } \\
\text { rate }\end{array}$ \\
\hline From office to the main restaurant & $75 \mathrm{~m}-150 \mathrm{~m}$ & 437 & 415 & $5.0 \%$ \\
\hline
\end{tabular}

Table 5. Path loss and standard deviation from different locations.

\begin{tabular}{lcc}
\hline Location & Average path loss & $\sigma$ \\
\hline Office (4th floor) & $97.8 \mathrm{~dB}$ & $8.40 \mathrm{~dB}$ \\
Bathroom (4th floor) & $97.2 \mathrm{~dB}$ & $7.80 \mathrm{~dB}$ \\
Coffee room (4th floor) & $100.4 \mathrm{~dB}$ & $6.36 \mathrm{~dB}$ \\
Main restaurant (below street level) & $109.1 \mathrm{~dB}$ & $10.51 \mathrm{~dB}$ \\
Main library (1st floor) & $133.7 \mathrm{~dB}$ & $8.58 \mathrm{~dB}$ \\
Guild room (below street level) & $146.1 \mathrm{~dB}$ & $4.95 \mathrm{~dB}$ \\
\hline
\end{tabular}

\subsection{Coverage with spreading factor 7}

As one can see, based on the results presented in Section 4.1, whilst operating with the maximum $S F$, the single base station covered all the measurement locations. This motivated us to investigate the coverage with the lower SFs. For this we have conducted a set of measurements at the 23 reference points (pointed with $\mathrm{dBm}$ values in Fig. 4) 
distributed around the campus area. The result for the $S F=7$ and $B W=125 \mathrm{kHz}$ are shown in Fig. 4 as a 3D radio signal heat map (created with Google Maps JavaScript API). As can be seen, the north part (top of figure) of the campus is not fully covered. Also it can be noted that the level of the signal received from the most western area of the campus is quite low and is close to the sensitivity level of the base station $(-124 \mathrm{dBm}$ for this DR setting). Also, as one can see from Fig. 4, the distance is not the only factor affecting the RSSI. In several cases (e.g., in the central area of the campus northwest from the base station) the closer points showed much higher attenuation of the signal. Please note that all the measurements were executed indoors and thus the outdoor points composing the heat map are the results of the extrapolation done by the used software and are only illustrative.

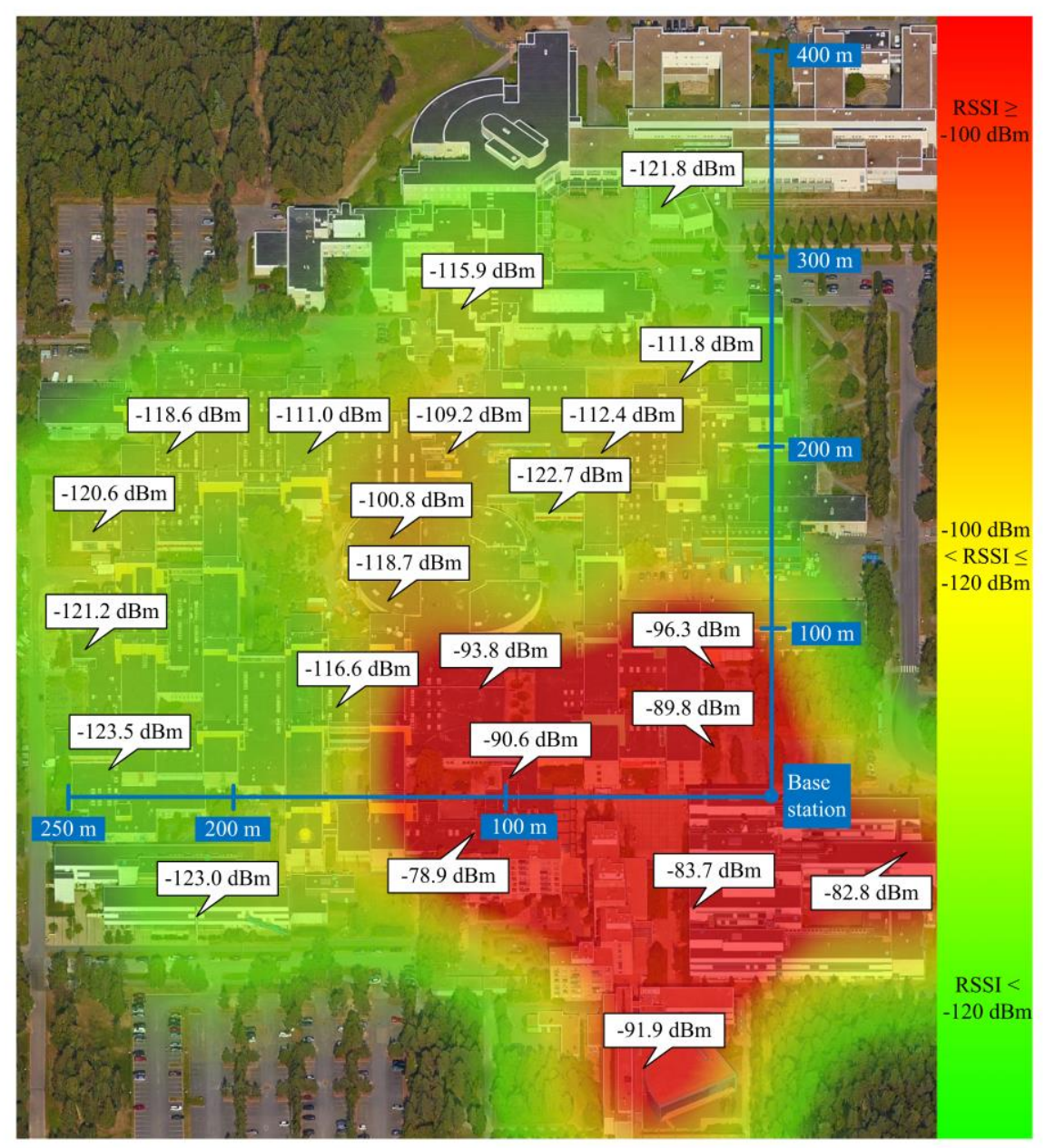

Fig. 4. Strength of the received radio signal from the different locations in University of Oulu $\left(P_{\mathrm{TX}}=2 \mathrm{dBm}, G_{\mathrm{RX}}=2 \mathrm{dBi}\right.$, air bit rate $\left.=5468 \mathrm{bps}\right)$. 


\subsection{Effect of the different SF}

The presented results in the two previous sections illustrate the performance of the LoRaWAN indoor communication for the minimum and maximum $S F$ s possible. This makes it easy for one to expect that the other $S F$ s should feature coverage and performance in between these two extreme cases. In order to verify this, we have conducted a set of measurements with the other $S F$ s. The total number of packets transmitted with the $S F=7,8,9$, and 10 was around 55000. Even though the number of packets seems reasonable for statistical analysis, only around 500 to 600 packets were transmitted per location and certain configuration. Certainly more would have given more reliable results, but unfortunately the low data rate and the harsh duty cycle restrictions imposed by the frequency regulations made the measurements very time consuming. Nonetheless, we think that the presented results provide useful information about the capabilities and limitations of the LoRa technology.

Table 6 shows the packet error rate (PER) and the average RSSI from 12 selected locations that are distributed between $55 \mathrm{~m}$ and $370 \mathrm{~m}$ distance away from the base station. The results show that only with $S F=7$ and $B W=125 \mathrm{kHz}$, the furthest location (library) was not reached. This result is rather surprising, given that using the same $S F$ and $B W=250 \mathrm{kHz}$ over $60 \%$ of the packets were received correctly.

Not surprisingly, the major packet losses occurred when the RSSI was at the edge of the radio sensitivity limit. For the signals exceeding $-100 \mathrm{dBm}$, the PER was well under $5 \%$. The presented results also reveal that the reliability of the connection for the different $S F$ s varies significantly. For example, from $205 \mathrm{~m}$ distance of the base station with $S F=7$ and $B W=125 \mathrm{kHz}$ the PER is $7.4 \%$, while for $S F=7(B W=250 \mathrm{kHz}), S F=8,9$ and 10 the PER is $57.2 \%, 20.7 \%, 8.4 \%$ and $2.5 \%$, respectively. There are many reasons which may have caused this behavior. First, the measurements with different configurations were done in different dates and time of day so the radio environment might have changed. Second, the number of transmitted packets is relatively small (500 to 600) as discussed above. Third, the position of the sensor node in each location might have shifted for the various experiments.

It is also worth noticing that few percent of the packets got lost regardless of the communication settings used even from relatively short distances. Data revealed that the lost packets were distributed uniformly over the entire measurement session, so packets were not lost in burst. In average, the number of packets lost in a row was slightly above one.

As discussed in Section 2.1, the access of a sensor to the communication media is done based on the frequency hopping spread spectrum algorithm, which pseudo randomly selects a frequency channel to be used, taking into the account the on-air time and the duty cycle restrictions imposed by the frequency regulations. Therefore, in the analysis of the experimental results we also investigated how the used channel affects the probability of successful data delivery. For this, we have characterized the distribution of all the received packets between the six used channels in two cases. In the first case, $99 \%$ of the packets were transferred successfully and the distribution is depicted in Fig. 5a. In the second case, the distribution (depicted in Fig. 5b) is obtained from measurement locations where more than $5 \%$ of the packets were lost. For both cases, around 6000 packets were accounted for. 
Table 6. Average packet error rate and received signal strength from different locations with different spreading factors.

\begin{tabular}{|c|c|c|c|c|c|c|c|c|c|c|}
\hline \multirow{3}{*}{$\begin{array}{l}\text { Distance to } \\
\text { the base } \\
\text { station }\end{array}$} & \multicolumn{4}{|c|}{$S F=7$} & \multirow{2}{*}{\multicolumn{2}{|c|}{$\begin{array}{c}S F=8 \\
B W=125 \mathrm{kHz}\end{array}$}} & \multirow{2}{*}{\multicolumn{2}{|c|}{$\begin{array}{c}S F=9 \\
B W=125 \mathrm{kHz}\end{array}$}} & \multirow{2}{*}{\multicolumn{2}{|c|}{$\begin{array}{c}S F=10 \\
B W=125 \mathrm{kHz}\end{array}$}} \\
\hline & \multicolumn{2}{|c|}{$B W=125 \mathrm{kHz}$} & \multicolumn{2}{|c|}{$B W=250 \mathrm{kHz}$} & & & & & & \\
\hline & $\frac{\sqrt{2}}{\frac{d}{a}}$ & 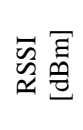 & $\frac{\sqrt{2}}{\frac{a}{2}}$ & $\begin{array}{l}\overline{\tilde{\sigma}} \\
\hat{\approx} \\
\approx\end{array}$ & $\frac{5}{\frac{2}{2}}$ & 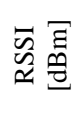 & $\begin{array}{l}\frac{2}{2} \\
\frac{1}{2}\end{array}$ & $\begin{array}{l}\overrightarrow{\tilde{\sigma}} \bar{\Xi} \\
\hat{\approx}\end{array}$ & $\frac{5}{\frac{5}{2}}$ & 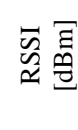 \\
\hline $55 \mathrm{~m}$ & 2.9 & -90 & 2.6 & -86 & 6.0 & -89 & 0.9 & -87 & 2.5 & -99 \\
\hline $65 \mathrm{~m}$ & 1.0 & -84 & 3.0 & -87 & 1.1 & -81 & 2.3 & -93 & 9.0 & -114 \\
\hline $95 \mathrm{~m}$ & 1.7 & -83 & 2.5 & -83 & 0.7 & -82 & 8.1 & -98 & 1.2 & -90 \\
\hline $165 \mathrm{~m}$ & 2.4 & -92 & 40.6 & -120 & 7.7 & -102 & 9.5 & -103 & 6.8 & -102 \\
\hline $180 \mathrm{~m}$ & 21.1 & -119 & 27.3 & -120 & 5.4 & -102 & 14.3 & -120 & 4.4 & -114 \\
\hline $195 \mathrm{~m}$ & 3.8 & -101 & 13.7 & -110 & 7.3 & -101 & 7.1 & -105 & 1.9 & -107 \\
\hline $205 \mathrm{~m}$ & 7.4 & -112 & 57.2 & -123 & 20.7 & -123 & 8.4 & -107 & 2.5 & -102 \\
\hline $215 \mathrm{~m}$ & 25.7 & -123 & 79.0 & -124 & 63.4 & -129 & 12.3 & -124 & 23.3 & -130 \\
\hline $230 \mathrm{~m}$ & 5.1 & -109 & 15.9 & -112 & 3.8 & -100 & 13.0 & -130 & 1.9 & -97 \\
\hline $305 \mathrm{~m}$ & 13.7 & -119 & 32.0 & -119 & 15.5 & -122 & 69.2 & -132 & 72 & -135 \\
\hline $310 \mathrm{~m}$ & 12.6 & -116 & 19.7 & -113 & 7.7 & -109 & 17.4 & -118 & 67 & -124 \\
\hline $370 \mathrm{~m}$ & No & & 35.1 & -119 & 17.8 & -119 & 19.6 & -117 & 36 & -130 \\
\hline Average & 8.9 & -104 & 27.4 & -110 & 13.1 & -105 & 15.2 & -111 & 22 & -112 \\
\hline
\end{tabular}

As can be seen, the probability of receiving a packet on channels with center frequencies of $868.300 \mathrm{MHz}$ and $869.525 \mathrm{MHz}$ is somewhat lower than for the four other channels. This can be interpreted as that more packets have been lost in either of these two channels, which may have happened, e.g., due to the different antenna efficiencies or amplification gains for the frequencies, due to the interferences from other systems, or due to the differences in the radio frequency propagation for these frequency bands. Another interesting result is that the variance of the distribution for Fig. $5 b$ is 3.55 -fold higher than the one for Fig. 5a. Given that this information will be made available to a sensor (e.g., by means of acknowledgements or some other ways) the node may attempt to take the lossy channels out of use. 


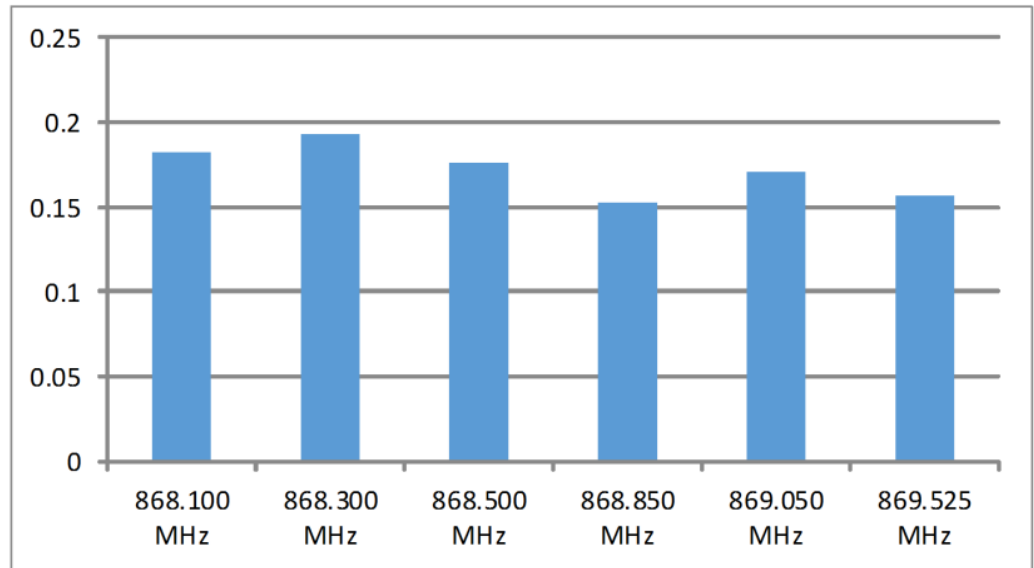

a)

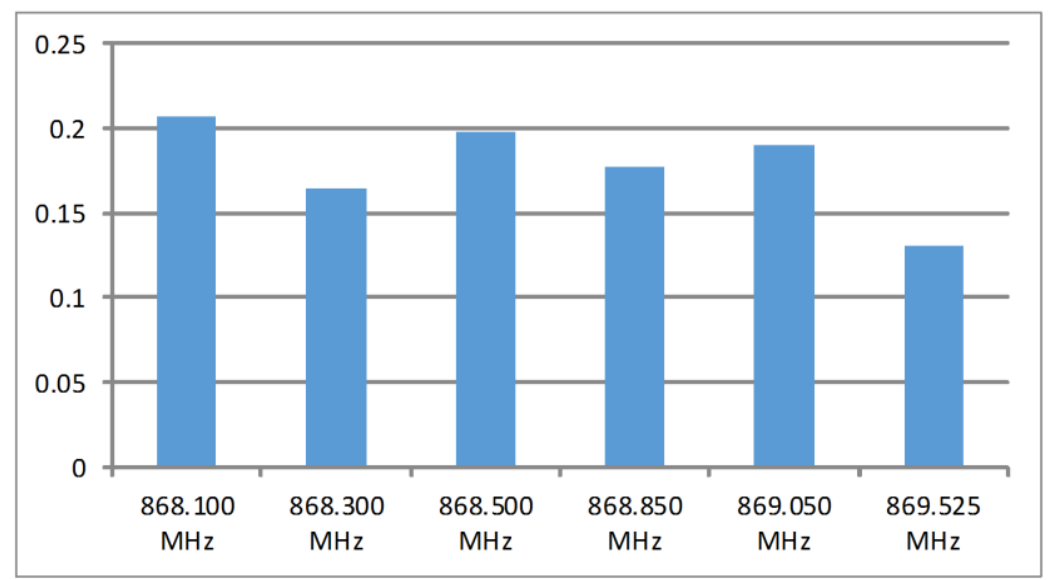

b)

Fig. 5. Probability of receiving a packet in different channels a) with $1 \%$ lost packets in total and b) total packet lost ratio over $5 \%$.

\subsection{Power consumption}

Aside of the coverage and the probability for successful radio packet's transmission, the DR used affects the on-air time and consequentially the amount of the energy consumed by a sensor node for sending a packet. Given that the majority of the human centric applications are developed based on mobile devices, the energy efficiency and battery lifetime becomes one of the primary concerns for these use cases. Due to this reason we also measured the amount of energy consumed for a radio packet transmission.

The measurements were done using our own LoRa module based on Microchip's RN2483 LoRa modem and integrated it into our modular wireless sensor and actuator network platform [23, 24]. For accurate power consumption measurements we used 
Keysight's N6705B DC power analyzer with the N6781A module. The measurement setup depicting LoRa module and the power analyzer are shown in Fig. 6. For the measurements the node was configured to send the radio packet with 16 byte application-level payload using the different DRs and transmit power settings. The power consumption profile was captured with the N6705B power analyzer and further processed.

The power consumption curves of the LoRa modem powered at $3.3 \mathrm{~V} \mathrm{DC}$ with GFSK and LoRa modulations using different $S F \mathrm{~s}$ and $B W \mathrm{~s}$ are depicted in Fig. 7. Fig. 7 essentially highlights two things. First, when the transceiver is transmitting at full power it consumes over $120 \mathrm{~mW}$. Therefore, to save the energy the transmission duration should be minimized. Second, the on-air time for the consecutive $S F$ roughly doubles. What is more than this, Fig. 8, which illustrates the effect of the transmit power on the power consumption, shows that the amount of energy consumed for the very same packet sent with the minimum and the maximum transmit power differs by more than $50 \%$. The further analysis of these data shows that the amount of energy required for sending the data using GFSK with $2 \mathrm{dBm}$ transmit power (resulting in the minimum energy consumption) and LoRa $B W=125 \mathrm{kHz}$ with $S F=12$ (resulting in the maximum energy consumption) is $0.57 \mathrm{~mJ}$ and $113 \mathrm{~mJ}$, respectively. The LoRaWAN specification defines six possible transmit power levels: 2, 5, 8, 11, 14 and $20 \mathrm{dBm}$. This emphasizes the importance of using the LoRa ADR feature in the energy-limited applications.

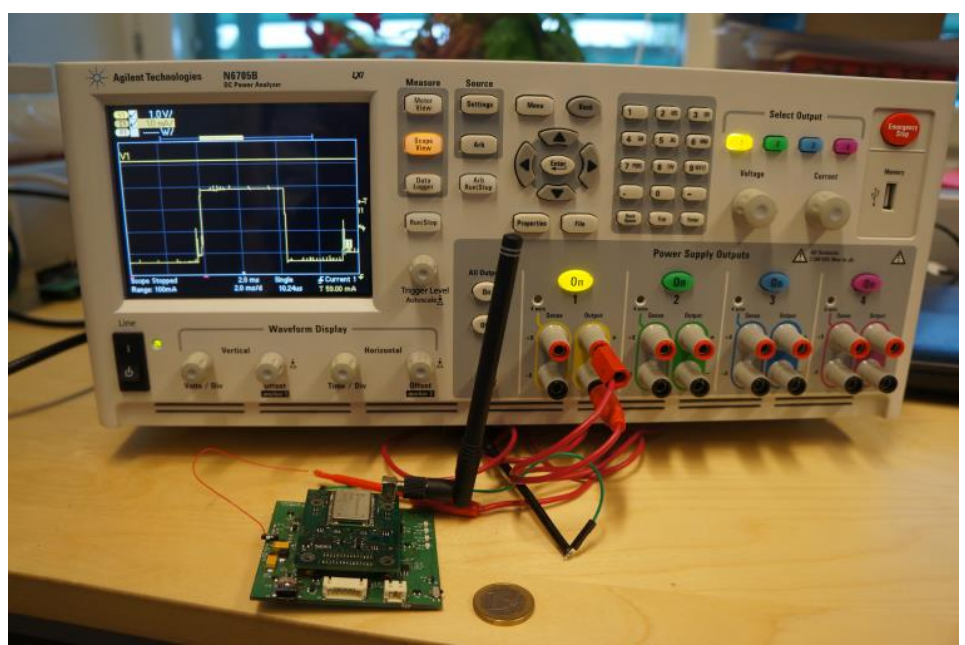

Fig. 6. Power consumption measurement setup for RN2483 LoRa modem. 


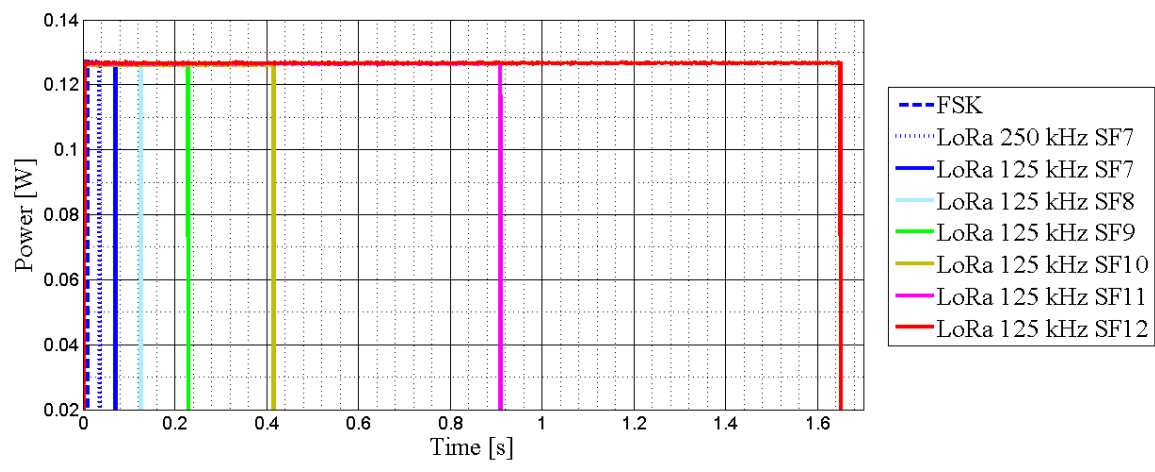

Fig. 7. Power consumption profile for 16-byte packet transmission whilst using the different LoRaWAN DRs (Ptx=14 dBm).

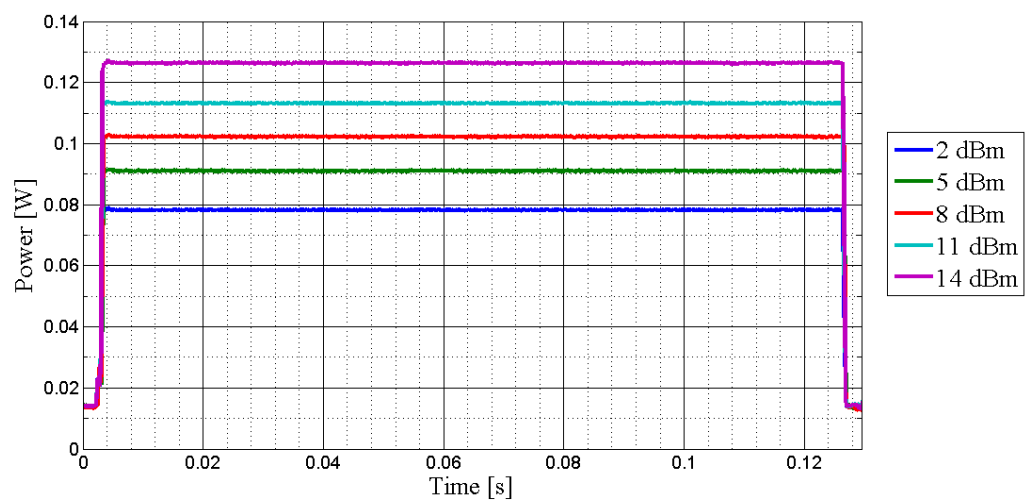

Fig. 8. Power consumption for 16-byte packet transmission utilizing different transmit power levels (LoRa modulation, $B W=125 \mathrm{kHz}, S F=8$, packet size $=16$ bytes).

\section{Conclusions}

To the best of our knowledge, this paper represents one of the first attempts to practically evaluate the feasibility of using the recently developed LPWAN technologies in the indoor environment in the context of human-centric applications. To this end, in the paper we have reported the results of an extensive measurement campaign conducted in the premises of the University of Oulu which give a hint about the coverage and the reliability of the LoRaWAN communication in the real-life environment. In the paper we first review the important technical details of the physical layer, medium access and network architecture of the LoRaWAN technology. Then we 
report the results of the measurements which have been conducted in two stages First, we investigated and characterized the performance of the LoRa communication with largest spreading factor of 12 and highest transmit power of $14 \mathrm{dBm}$ while used for monitoring researcher's well-being in a workplace during typical work days. The measurement results have proven the potential of using the LoRaWAN for these applications and revealed that for all the tested locations over $96 \%$ of the packets sent by the sensor node were successfully received by the single base station. Therefore in the second phase, we measured indoor coverage with lower spreading factors featuring shorter on-air time.

The measurements were conducted using a commercial sensor node equipped with Semtech's SX1272 LoRa compliant transceiver. The antenna of the base station was located on the antenna tower at $24 \mathrm{~m}$ height at the roof of Faculty of Information Technology and Electrical Engineering building, University of Oulu, Finland. The obtained results show that the LoRa technology provides sufficiently good coverage even with lower spreading factors when operating in difficult locations from the radio signal propagation point of view. Nonetheless, it was noted that the communication performance is subject to the strong variations and is affected by both the link's distance and the materials and obstacles blocking the link.

Aside of this, we have conducted the measurements of the power consumption of the LoRa compatible radio transceiver operating with different modulations, bandwidths, spreading factors and transmit powers. For this we integrated a LoRa compliant transceiver into our modular wireless sensor and actuator platform and and measured the respective consumption profile using a highly accurate power analyzer. The results clearly illustrate the difference in on-the-air times and the consumed energy for different configurations, and emphasize the importance of deliberated selection of the radio's operation mode and the use of the adaptive data rate feature for optimizing the data rate and transmit power for the sensor node.

Based on the presented results, we can draw the following conclusions. First, we have experimentally confirmed the feasibility of using the LPWANs, and specifically the LoRaWAN, for establishing indoor communication in general, and for the human-centric applications in particular. Second, we have proved that the indoor communication links of over 300 meters are possible. Third, we have noticed that the performance of the indoor communication is not perfectly stable and may vary significantly depending on the communication settings used. Importantly, we have witnessed that not always the maximum spreading factor gives the most stable communication link. This is worth remembering when developing an algorithm for ADR. Fourth, we have seen that even for the sufficiently short distances at least few percent of packets get lost regardless of the communication settings used. What is more than this, the transmission of a single LoRaWAN packet depending on the communication mode settings takes from few milliseconds and up to few seconds. This makes this technology more suitable for the delay and loss tolerant applications rather than the dependent applications imposing strict quality of service requirements (as many smart healthcare applications do). Even though, the LoRaWAN technology can be potentially used for the distributed wellbeing monitoring and control of the 
non-critical signs, such as the physical activity or location tracking, monitoring of pets and animals, various vocational wellbeing and staff management applications, etc.

As a future work, we plan to conduct similar measurements with the other LPWAN technologies, such as Sigfox, LTE-M, Weightless, etc. and compare their performance with the results reported in this paper. Additionally, we plan to continue our work with the LoRaWAN technology and investigate how tolerant is the technology towards the interferences.

Acknowledgements. The authors wish to thank Marko Pettissalo from Nokia for providing the commercial LoRa sensor node and the base station used in this study. This work has been partially funded by the Finnish Funding Agency for Innovation (Tekes) through VIRPA C project.

\section{References}

1. N. Hunn: LoRa vs LTE-M vs Sigfox. Available online: http://www.nickhunn.com/lora-vs-lte-m-vs-sigfox/

2. S. Movassaghi, M. Abolhasan, J. Lipman, D. Smith, A, Jamalipour: Wireless Body Area Networks: A Survey. IEEE Comm. Surveys \& Tutorials, vol. 16(3), (2014)

3. J. Petäjäjärvi, K. Mikhaylov, R. Vuohtoniemi, H. Karvonen, J. Iinatti: On the Human Body Communications: Wake-up Receiver Design and Channel Characterization. EURASIP Journal on Wireless Comm. and Netw., 2016:179, (2016)

4. E. Kartsakli, A. Lalos, A. Antonopoulos, S. Tennina, M. Di Renzo, L. Alonso, C. Verikoukis: A survey on M2M systems for mHealth: A wireless communications perspective. Sensors, vol. 14, pp. $18009-18052$, (2014)

5. S. Ullah, H. Higgins, B. Braem, B. Latre, C. Blondia, I. Moerman, S. Saleem, Z. Rahman, K. Kwak: A comprehensive survey of wireless body area networks - On PHY, MAC, and Network Layers Solutions. Journal of Med. Syst., vol. 36, pp. 1065 1094, (2012)

6. R. Sanches-Iborra, M. D. Cano: State of the Art in LP-WAN Solutions for Industrial IoT Services. Sensors 16(5):708, (2016)

7. A. Augustin, J. Yi, T. Clausen, M. Townsley: A Study of LoRa: Long Range \& Low Power Networks for the Internet of Things. Sensors 16(19):1466, (2016)

8. C. Goursaud, J. M. Gorce: Dedicated Networks for IoT: PHY/MAC State of the Art and Challenges. EAI Endorsed Trans. on Internet of Things (2015)

9. K. Mikhaylov, J. Petäjäjärvi, T. Hänninen: Analysis of the Capacity and Scalability of the LoRa Wide Area Network Technology. In: European Wireless Conf., Oulu, Finland, 18 - 20 May (2016)

10. J. Petäjäjärvi, M. Pettissalo, K. Mikhaylov, A. Roivainen, T. Hänninen: On the coverage of LPWANs: Range Evaluation and Channel Attenuation Model for LoRa Technology, In: ITS Telecommunications, Copenhagen, Denmark, 2 - 4 Dec. (2015)

11. J. Petäjäjärvi, K. Mikhaylov, M. Pettissalo, J. Janhunen, J. Iinatti: Performance of a LPWAN based on LoRa Technology: Doppler Robustness, Scalability and Coverage. Conditionally accepted to DWSN.

12. J. Petäjäjärvi, K. Mikhaylov, M. Hämäläinen, J. Iinatti: Evaluation of LoRa LPWAN Technology for Remote Health and Wellbeing Monitoring. In: ISMICT, Worcester, MA, USA, 21 - 23 Mar. (2016)

13. LoRa Alliance: LoRaWAN Specification v.1.0. Jan. (2015) 
14. Semtech: LoRa modulation basics - AN1200.13, Revision 1., Jan. (2015)

15. T. S. Rappaport: Wireless Communications - Principles and Practices. (IEEE Press, New Jersey, 1996)

16. Semtech: SX1272/73 - $860 \mathrm{MHz}$ to $1020 \mathrm{MHz}$ Low Power Long Range Transceiver. Datasheet, revision 3, Mar. (2015)

17. Semtech: SX1272/3/6/7/8 LoRa Modem Design Guide - AN1200.13, Revision 1, July (2013)

18. A. S. Tanenbaum. Computer Networks: The Medium Access Sublayer, 3rd edn., (Prentice-Hall, New Jersey, 1996), pp. 243 - 338

19. LoRa Alliance: A Technical Overview of LoRa and LoRaWAN, White paper, Nov. (2015)

20. Semtech: LoRaMote, Revision 2.0, July (2014)

21. Finnish Communications Regulatory Authority: 15AH/2015M - Regulation on Collective Frequencies for License-exempt Radio Transmitters and on Their Use. Feb. (2015)

22. Aerial: Biconical Antenna D100-1000.

23. K. Mikhaylov, J. Petäjäjärvi, M. Mäkeläinen, A. Paatelma, T. Hänninen: Demo Modular Multi-radio Wireless Sensor Platform for IoT Trials with Plug\&Play Module Connection. In: MobiCom, pp. 188-189, Sept. 7 - 12 (2015)

24. K. Mikhaylov, J. Petäjäjärvi, M. Mäkeläinen, A. Paatelma, T. Hänninen: Extensible Modular Wireless Sensor and Actuator Network and IoT Platform with Plug\&Play Module Connection. In: IPSN, pp. 386-387, Apr. 13 - 16 (2015) 